\title{
Aspectos tecnológicos de la reconversión industrial ${ }^{\star}$
}

\author{
Joaquín Arriola
}

Las estrategias de desarrollo industrial se suelen entender, como todas las estrategias de desarrollo, en términos de: intervención estatal (estrategias orientadas al mercado o a la planificación), el papel del comercio internacional (estrategias sustitutivas de importaciones $u$ orientadas a la exportación), la inversión (orientación hacia las industrias básicas o industrias de consumo final), y de perspectiva (rentabiliad del capital o perspectiva de necesidades básicas).

Dado que no existe consenso sobre cuales sean los aspectos importantes y sobre un orden de prioridades, es muy dificl hablar de una "política industrial", y menos de las estrategias más adecuadas para cada pals en función de la peculiar combinación de circunstancias que definan su situación particular.

En los últimos tiempos se está argumentando desde diversas instancias sobre la necesidad de una "reconversión industrial" de la manufactura de los palses centroamericanos, como la mejor estrategia para hacer viable el desarrollo de la base de las actividades industriales presentes y futuras.

En la definición de lo qùe es un proceso de reconversión industrial, nos encontramos con dos perspectivas radicalmente opuestas, en fun-

- Este articulo es una versión, ampliamente modificada, de la ponencia presentada en representación del Departamento de Economla de la UCA en el VII Congreso de pr Profesionales en Ciencias Económicas, sobre "Reconversión Industrial", San Salvador 29-31 de agosto de 1991. 
ción de la definición pasiva o activa de esta política.

Para unos, la reconversión industrial coincide con el proceso de renovación permanente de las empresas, poniendo un énfasis especial en la adaptación en un contexto cambiante en lo que a política arancelaria y política industrial se refiere: en particular, en un contexto de desregulación acelerada, con reducción general de tarifas a la importación, reducción de precios subsidiados a la industria y de exenciones fiscales o ventajas financieras.

En esta definición, que coincide con la idea que manejan de la reconversión los sectores neoliberales del país (FUSADES, Ministerio de Economía...) no se acierta a dotar de contenido específico al concepto de reconversión, que se confunde con modernización industrial, o con adaptación defensiva ante las modificaciones de precios relativos y de estructura de especialización.

Una definición activa de la reconversión industrial, basada en las experiencias de reconversión de los paises de la Comunidad Económica Europea (A. Hualde 1985) insiste por el contrario en las medidas de cambio estructural, a través de las distintas modalidades de intervención social en la economia: planificación macroeconómica, concertación, control público de sectores productivos. La reconversión es un mecanismo sofisticado de intervención en la economia, que trata de combinar las medidas de adaptación a nivel émpresarial o microeconómico, y a nivel sectorial o mesoeconómico, con un cuadro macroeconómico orientado por los objetivos cuantitativos en que se enmarca la reconversión.

En esta segunda perspectiva, diriamos que la reconversión industrial es una propuesta de intervención en la estructura económica, que resulta de un determinado diagnóstico de los problemas de una economla: cuando se considera que los males de la economía derivan de la crisis de la estructura productiva, y las políticas a aplicar no pueden ser solamente macroeconómicas y de corto plazo, sino que deben incidir en la transformación de la estructura económica. En este sentido, la reconversión industrial no puede ser complementaria de otras medidas, sino que implica una intervención más profunda que la simple reforma del sector público, la disciplina financiera o los acuerdos sociales en materia de distribución.

La reconversión industrial, piedra angular de una reforma en profundidad del sistema productivo, es un proceso complejo, que necesariamente se enfrenta a la manifestación de intereses múltiples y a veces contrapuestos de diversos actores sociales (Estado, trabajadores, capital productivo nacional, capital extranjero, capital financiero, sectores pro- 
ductivos...), que necesitan ser conciliados de algún modo, en torno a unos objetivos mínimos comunes compartidos.

Por otro lado, la reconversión no se da al margen de un contexto social e histórico concreto. En la región centroamericana, en la era de la tercera revolución industrial, caracterizada por la aplicación productiva de la economía del tiempo y la mundialización de los procesos productivos, la reconversión industrial debe adoptar pertiles propios, cuyo diseno está todavia muy lejos de haberse completado.

En el caso de El Salvador, la reconversión no puede limitarse a intervenir sobre la capacidad instalada, sino que necesariamente debe incluir un conjunto de propuestas viables para ampliar la base productiva del pais, diversificar la actividad manufacturera y relanzar la producción del sector.

\section{Contenldos de la reconversión Industrlal}

Para tener una perspectiva más ajustada de lo que significa la reconversión industrial, vamos a desagregar los diferentes aspectos económicos y sociales que debe incluir un proceso de intervención sectorial de esa dimensión.

Siguiendo a M. Navarro (1989), podemos identificar seis elementos que deben ser tomados en consideración a la hora de disenar una política de reconversión:

1) El engarce de la política de reconversión con la politica economica general y con la politica Industrlal

Es evidente que toda empresa o sector en reconversión será afectado por el tipo de política macroeconómica que se impulse desde el gobierno; una política económica que, por ejemplo, dé lugar a altas tasas de interés, tendrá un efecto claramente negativo en empresas caracterizadas por una elevada proporción de recursos ajenos en sus pasivos, situación .bastante frecuente entre las empresas y sectores necesitados de una reconversión. Por el contrario, una política económica que favorezca la reactivación, influye favorablemente en las empresas y sectores en reconversión, al posibilitar una mejora en las ventas e inversiones, al incrementar la demanda y reducir el coste de la financiación del capital. El entorno macroeconómico debe cumplir algunos requisitos antes de poder viabilizar cualquier plan de reconversión. En particular, los recursos del Estado deben estar suficientemente garantizados para hacer frente al coste financiero de la reconversión. No se puede esperar que toda la financiación provenga del exterior, por lo tanto una reforma 
fiscal, que situe en las rentas personales, y las rentas del capital las principales fuẹtes de financiación de la Administración, es un ineludible paso previo a la implementación de la política de reconversión.

Es igualmente importante que la politica de reconversión se enmarque en una política industrial más amplia, que junto a los ajustes en cantidades y calidades de la oferta, incentive la reindustrialización y la ampliación de las oportunidades de inversión productiva, ampliando las actividades y con un tratamiento de promoción sectorial y selectiva de las actividades de futuro.

\section{2) El grado de concentración y particlpación soclal}

Sin la participación en la elaboración de la política de reconversión de todos los agentes implicados (empresas/patronal, trabajadores/ sindicatos, Administración, banca, partidos políticos, gobiernos locales) dificilmente se logrará la aceptación de los sacrificios que ineludiblemente van aparejados a este tipo de políticas. La reconversión sin concertación es impensable, salvo a costa de un deterioro social muy fuerte. Esto es especialmente relevante en lo que respecta a los que sufren más las consecuencias del ajuste: los trabajadores y los sindicatos. Estos solo aceptarán asumir los probables costes de la reconversión (disminución del empleo, cierre de empresas, deterioro de los niveles salariales...) a cambio de las adecuadas compensaciones (reciclaje profesional, seguro de desempleo, participación en las ganancias futuras de productividad etc.). En definitiva, las compensaciones sólo se pueden garantizar para los trabajadores si, paralelamente a la negociación del ajuste, se negocia la reindustrialización.

En todo caso, también hay que reconocer la posibilidad de consensos entre empresarios y trabajadores que vayan en contra de la viabilladd de la polltica de reconversión, por ejemplo oponiendose al cierre de empresas o a la concentración de empresas con reducción de empleo y pérdida del control por parte del empresario individual. En tales casos, la Administración debe situar el cumplimiento de los objetivos (pactados) generales de la reconversión por encima de los desacuerdos sectoriales o puntuales en una u otra empresa.

3) El horizonte Industrlal y el ámblto de aplicación de la polftica de reconversión

Dado que la politica de reconversión es solo la primera fase de ajuste, pero que ineludiblemente debe estar acompanada de otra de reindustrialización y mejora del entorno tecnológico, la reconversión debe ir enmarcada en un plan de actuación a medio plazo que fije los objetivos 
generales de la política industrial, en materias tan diversas como tasas de penetración en mercados regionales y de ultramar, tasas de inversión, objetivos de concentración y desconcentración sectorial y territorial etc.

4) Los procedimlentos de elaboraclón, aprobación, gestlón, control y segulmlento de los planes

La eficacia de la política depende en gran medida de la capacidad técnica y burocrática que se demuestre en su diseño, implementación y evaluación. Los supuestos en los que se basan los planes de reconversión deben ser realistas: la planificación de la evolución de la demanda actual y potencial, la capacidad de absorción del cambio técnico y la rapidez de la adaptación al cambio de trabajadores y empresarios son elementos que, si no están adecuadamente calibrados, pueden orientar equivocadamente los planes que se elaboren.

La transparencia en todas las fases del proceso, desde la selección de sectores y empresas hasta la asignación de beneficios financieros u otros, pasando por los criterios para el establecimeinto de prioridades y los criterios selectivos, es un aspecto imprescindible para lograr el grado de consenso nacional requerido por un plan de esta envergadura.

\section{5) Los tlpos de medldas aplicadas}

Las medidas básicas de todo plan de reconversión son de tres tipos:

a) Fiscales: bonificaciones en impuestos, libertad de amortización de activos, deducciones por inversiones, fraccionamientos y aplazamientos de las deudas tributarias etc. Son las menos recomendables, por su escasa transparencia y coste desconocido, que las vuelven poco eficaces, además de dificultar la puesta en marcha de la reforma fiscal.

b) Financieras: subvenciones, créditos oficiales, avales públicos, aportaciones públicas de capital... la participación del sector privado es imprescindible para complementar las medidas financieras públicas, y lograr que estas consigan sus objetivos. La iniciativa privada puede colaborar con un conjunto muy amplio de medidas finacieras: préstamos participativos, coeficientes de inversión sectorial obligatoria, tasas de interés preferentes, etc.

c) Laborales. Estas medidas se centran por un lado en el tratamiento unitario de las condiciones de trabajo y niveles de empleo en los procesos de fusión, segregación, agnupación o absorción de empresas, y en el establecimeinto de mecanismos de protección extraordinarios destinados a amortiguar los efectos del ajuste de plantillas: jubilaciones 
anticipadas, fondos de promoción de empleo y otras que se pudieran diseñar eventualmente.

\section{6) El coste de los recursos públicos absorbldos por la reconversión}

Sin una adecuada contabilización del coste actual e indirecto de las medidas adoptadas para llevar adelante el plan de reconversión, se hace muy dificil el seguimiento de su aplicación y la evaluación de los resultados. En este sentido, en conveniente dar a los fondos recibidos del exterior el tratamiento de recursos públicos propios, y evaluar convenientemente el coste de oportunidad de cada una de las medidas que se propongan.

\section{El momento tecnológlco de la reconversión}

Plantear la reconversión industrial en 1991 desde El Salvador requiere de un cuidadoso análisis previo de las tendencias de los procesos productivos a escala mundial, para identificar los espacios posibles de actividad productiva nacional a ampliar o implementar, y los requisitos que esto conllevaria. Además, el imprescindible incremento de la productividad, inherente a todo proceso de reconvérsión industrial, es aun más importante en el caso de los paises centroamericanos, por la escasa productividad de sus sector manufacturero, comparativamente con la de otras regiones desarrolladas y subdesarrolladas: salvo en el caso del Africa subsahariana y de Indochina, ninguna región o subregión del planeta presenta niveles tan reducidos de crecimiento de la productividad del trabajo en la manufactura.

Muchas industrias en la mayoria de los paises subdesarrollados no han madurado en el sentido de haber alcanzado una producción internacionalmente competitiva, a causa de la ausencia de un esfuerzo sostenido para adquirir y usar las capacidades necesarias para lograr un cambio tecnológico continuo. En una fase de aceleración del cambio tecnológico, como es la que vivimos actualmente, la progresiva pérdida de dinamismo del sector manufacturero se transforma en crisis abierta.

La actual fase de desarrollo capitalista se caracteriza por la acelerada transformación tecnológica de los procesos productivos. La base de este cambio se encuentra en las tecnologia de la información (microelectrónica), que están revolucionando toda la actividad económica, redisenando el propio concepto de relaciones económicas internacionales y los espacios productivos nacionales. Así, el incremento de la productividad debemos verlo, en el nivel de las actividades productivas individuales (empresas) en términos de cambio tecnológico. 
Actualmente, las tendencias del cambio tecnológico están dominadas por la actividad económica de las firmas multinacionales, cuya estrategia de desarrollo se apoya en:

a) La fragmentación de los procesos productivos a escala mundial, posible gracias a las nuevas tecnologias que reducen el número de componentes y automatizan aceleradamente las fases de composión de productos finales (especialización flexible).

b) La existencia de nuevas facilidades para acceder a recursos financieros (desregulación bancaria, unificación de los mercados financieros internacionales).

c) El uso de nuevas técnicas de organización del trabajo (circulos de calidad, descentralización y participación en la toma de decisiones) que aumentan la productividad del trabajo.

d) Nuevas estategias de compras y de fabricación (subcontratación) que dotan de un nuevo contenido a los conceptos de calidad del producto y ayudan a la reducción de los inventarios (just-in-time), aumentando la capacidad de autofinanciación de las empresas.

Estas transformaciones en las fuerzas productivas están volviendo inadecuada la interpretación tradicional de la actividad económica: hoy menos que nunca, las "ventajas comparativas" no son un concepto adecuado para interpretar la competitividad internacional, que por el contrario se caracteriza a partir de los rendimientos crecientes, que derivan de la velocidad de adaptación de una economia a los cambios del mercado, su capacidad endógena de innovación de productos y procesos, la competencia por la calidad etc., de modo que es la flexibilidad y no el coste de los factores el factor determinante de la competencia internacional.

Las nuevas características de los procesos productivos están modificando igualmente la orientación geográfica de la inversión industrial, y alterando los criterios de rentabilidad y coste: el precio de la fuerza de trabajo es cada vez menos determinante de la localización de la inversión industrial, incluso en industrias intensivas en mano de obra, pasando a jugar un papel más relevante la cercania a los mercados, el acceso a las fuentes de información aplicable en el proceso de producción, las rentas de situación derivadas de la proximidad a otras industrias complementarias, tanto de producción como de servicios, la capacidad del Estado de responder a los retos de inversión pública que requiere la constante modificación de las variables inciorporadas al proceso productivo (formación, infraestructura y tecnologla de información) y su política de incentivos a la inversión etc. 
En este proceso, se modifica también el rol de los agentes económicos; por ejemplo, el diferente comportamiento internacional de las economias de América Latina frente a la de los nuevos paises industrializados del sudeste asiático deriva del distinto papel jugado por el Estado y las reformas estructurales y los capitales nacionales en una y otra región.

El estancamiento industrial de América Latina, cuyo grado de industrialización en 1983 era equivalente al de 1970, contrasta con el dinamismo industrial del sudeste asiático: las exportaciones de manufacturas, que duplicaron su importancia en las exportaciones totales de la periferia entre 1970 y 1984 ( del 14\% al 30\%), mostraron un signo muy diferente en una región y en otra: mientras que los paisess del sudeste asiático lograban aumentar su cuota del mercado manufacturero de los paises desarrollados del $3 \%$ al $10 \%$, América Latina solo alcanza una cuota del $2 \%$ en ese mismo mercado (J. Knakal 1989, pp.8-9).

La explicación principal de este diferente comportamiento no se encuentra en una diferencia de orientación exportadora o sustitutiva de importaciones, sino en el papel activo del Estado, en Corea y Taiwan, en promover la industrialización interna y la adaptación tecnológica, identificando actividades y planificando la diversificación sectorial, controlando la inversión extranjera para facilitar la absorción nacional de la tecnología, realizando reformas agrarias que internalizaron los precios relativos de reproducción de la fuerza de trabajo, disminuyendo el coste relativo de la fuerza de trabajo por la via de la mejora de la productividad agricola, con inversiones en formación de la mano de obra (el trabajador industrial medio en Corea tiene un nivel de formación de bachiller) que incrementaron grandemente la adaptabilidad de esta al conocimiento de nuevos procesos de trabajo y nueva maquinaria, y una política selectiva de protección de las industrias nacientes mediante barreras arancelarias y contingentación, créditos preferentes y ventajas fiscales y de fomento de la competencia en las industrias maduras, abriéndolas al comercio internacional (H. Pack y L. Westphal 1986 y J. Arriola 1989).

Es evidente que una intervención selectiva tan compleja solo puede dar resultados aceptables en un contexto de aplicación y reformulación permanente de los detalles de la estrategia de intervención, mediante la acumulación de información relevante que permita juzgar los avances parciales hacia el objetivo concreto del desarrollo industrial. Concebida la industrialización en términos de cambio tecnológico, el esfuerzo requerido se orienta a "usar la información tecnológica y a acumular conocimiento tecnológico para evaluar y elegir tecnologia, para adquirir y 
operar procesos y producir productos, gestionar cambios en los productos, procesos, procdedimientos y criterios organizativos; y para crear nueva tecnología" (Pack y Westphal 1986, p.105).

El proyecto de reconversión industrial tiene que partir necesariamente de la elección de un modelo tecnologico y así, la reconversión industrial es necesariamente un proceso de inversión en capacidad tecnologica, que es la habilidad de hacer un uso efectivo del conocimiento tecnológico,por parte de la economia en su conjunto y de las empresas, el Estado y los trabajadores de modo específico, al ser estos los agentes, sujetos y objetivo de la reconversión industrial.

Las tecnologias para industrias de reciente implantación, o con capacidad instalada obsoleta desde el punto de vista técnico, no puede ser absorbida sin cambios tecnológicos adaptativos o incrementales, con lo cual decimos que el cambio tecnológico es un proceso permanente, que requiere un aprendizaje para su continuidad temporal. Pero este aprendizaje dificilmente se va a lograr con actuaciones que incidan solo desde y sobre las actividades productivas individuales.

En esta perspectiva, el problema del Estado no es su grado de intervención, sino el de la calidad de la intervención; no se trata de la reducción de la actividad económica del Estado, sino de su reformulación y recualificación. En un pais con una escasa e ineficiente base industrial, las concepciones ideológicas basadas en el principio de "menos Estado" es incompatible con la adquisición y difusión al tejido productivo nacional de las capacidades tecnologicas de la industria moderna.

Una fuerza de trabajo con escaso nivel de formación es una rémora para el aumento de la capacidad tecnólogica. Los cambios acelerados en los procesos de trabajo exigen un esfuerzo de formación permanente a cualquier pais que desee avanzar en el desarrollo. Pero la formación permanente no se puede generalizar alli donde no están cubiertas las necesidades básicas de la educación. Por eso es inadecuado interpretar la asignación de recursos a la educación de la población (o el gasto en sanidad) como un coste, cuando en realidad es una inversión a medio y largo plazo, imprescindible en la búsqueda da la transformación de la base productiva de la nación.

La reconversión industrial de las empresas plantea diversas dimensiones: la cooperación tecnológica con el capital extranjero, especialmente el multinacional, se debe plantear desde la Administración con las debidas cautelas, para garantizar la incorporación a las fuerzas productivas nacionales de las tecnologias incorporadas en la nueva actividad, sea realizada directamente por el capital multinacional en sueb 
nacional, $o$ en colaboración con empresas nacionales. Esto require un proceso de negociación permanente con las empresas multinacionales, que suponer tanto el consenso de los empresarios con la Administración, como la acción conjunta de diversos gobiernos, a escala regional y mundial.

La modificación de la organización del trabajo es parte esencial de la tercera revolución industrial, además de tener una fuerte influencia en la naturaleza del cambio tecnológico y por tanto en el comportamiento de aprendizaje (J. Katz 1984). La incorporación de las nuevas tecnologlas no se puede entender exclusivamente como la adquisición de nueva maquinaria, la fabricación de nuevos productos y la implementación de nuevos procesos: la transformación de las capacidades de dirección empresarial es uno de los retos más importantes de cualquier proceso de reconversión industrial. Esto implica no solamente la adquisición de nuevos conocimientos gerenciales o la aplicación de nuevas técnicas de gestión de la fuerza de trabajo, sino un completo cambio de mentalidad empresarial, que genere una nueva actitud hacia la innovación, la competencia y la colaboración interempresarial. Por lo mismo, la relación con el Estado no puede limitarse a la búsqueda de un trato preferencial. La consideración de la fuerza de trabajo exclusivamente en términos de coste, o de la fiscalidad sobre la actividad empresarial en términos de "apropiación indebida" por parte del Estado de lo que en otro caso sería parte de la ganancia del capital, no favorece tampoco esta reconversión empresarial: el esfuerzo por favorecer la cualificación y recualificación de la mano de obra, la reconsideración del papel de los servicios públicos de salud, educación y protección social en términos de inversión para la mejora de la eficiencia de la economia en su conjunto, la consideración del aporte productivo de la participación de los trabajadores en la empresa y en la sociedad, son otros tantos "intangibles" cuya desconsideración en la política de reconversión abonan el camino hacia el fracaso de esta.

La escasa comprensión teórica de la relación existente entre cambio tecnológico y desarrollo industrial se ha traducido en que las políticas de ampliación de las actividades manufactureras se hayan basado más en la sobreprotección y el paternalismo estatal que en una adecuada combinación de protección de las industrias nacientes y apertura para las que entran en fase de maduración, y sin los incentivos necesarios para incrementar la absorción tecnológica. La reacción aperturista que se observa en la política disenada por FUSADES/AID e implementada por el actual gobierno de ARENA es un desplazamiento al extremo opuesto, pero que tampoco refleja una adecuada comprensión de el fenómeno de la industrialización: reducir la intervención económica a una política de 
modificación de los precios relativos, y esperar que por generación espontanea las empresas adquieran la capacidad que hasta ayer se les desconocia para adaptar su proceso productivo a las condiciones dominantes en el mercado internacional, con la sola información de los nuevos precios, desconsidera el importante esfuerzo de difusión de información, de concertación social y de inversión sectorial de recursos financieros y humanos que requiere la reconversión del parque industrial del pais.

\section{REFERENCIAS}

Joaquín Arriola: Los nuevos paises industrializados. transferencias tecnológicas y subdesarrollo lepala, Madrid 1989.

Joaquín Arriola: "Sobre la reconversión industrial" en Realidad económico-social num.19 enero-abril 1991.

Alfredo Hualde: "Aspectos de las reconversiones industriales en Francia y España" en Mapa Económico Internacional num.4, CIDE, Mexico 1985.

Francisco Javier lbisate: "El modelo económico salvadoreño en la matriz insumo-producto: 1978", en Boletín de Ciencias Económicas y Sociales año IX num. 5, sept.-oct. 1986.

Jorge Katz: "Domestic technological innovations and dynamic comparative advantage" en Journal of Development Economics num.16 1984.

Jan Kñakal: "Las empresas transnacionales y los gobiernos frente a la transformación productiova en América Latina" en actas del III Congreso $\mathrm{Na}$ cional de Economla, La Coruña 7-10 de diciembre 1989.

Carlos Mackinlay: "Estructura productiva y tamaño de las economias: el caso de las pequeñas economlas latinoamericanas", en Economia de América Latina, num.15, 1986.

Mikel Navarro Arancegui: "La polltica de reconversión industrial en España" en Información Comercial_Española enero 1989 45-69.

H. Pack y L.E. Westphal: "Industrial Strategy and Technological Change. Theory versus Reality" en Journal of Development economics 221986 87-128.

Nathan Rosenberg: Tecnologla y economia Gustavo Gili, Barcelona 1986.

Julio Segura: "La crisis económica como crisis industrial: la necesidad de una estrategia activa" en Papeles de Economía num.15 1983. 